Un método para proyectar la población según tamaño de la localidad. (Aplicación al caso de la población urbana de México en 1990)

\title{
Virgilio Partida Bush*
}

Ante la carencia o la poca confiabilidad de los datos necesarios para hacer proyecciones de población al nivel de localidad —o de grupos de localidades - mediante el método de las componentes demográficas, se propone un método de las componentes restringido, en el sentido que no se considera sexo ni edad. El procedimiento consiste en descomponer el factor multiplicativo de crecimiento total en sus componentes de crecimiento natural y social, y proyectar cada una por separado. El método se ejemplifica con el caso de México para la década de los años ochenta, incluyendo la estimación y proyección de las componentes a partir de las cifras censales para 1960, 1970 y 1980.

\section{Introducción}

Las proyecciones de población constituyen una herramienta útil para la planeación económica y social, y su utilidad aumenta conforme es mayor el nivel de desagregación con que se obtienen. Entre los diferentes métodos disponibles para hacer proyecciones de la población, el llamado de las componentes es indudablemente el mejor, pues basado en previsiones de los niveles y tedencias por edad y sexo de las tres variables o componentes demográficas (fecundidad, mortalidad y migración), incorpora el mayor conocimiento adquirido sobre la dinámica demográfica. Sea por la carencia o por la poca confiabilidad en los datos necesarios, la inmensa mayoría de las aplicaciones del método de las componentes se ha hecho sólo al nivel nacional.

Un procedimiento alternativo ampliamente difundido para obtener futuros escenarios subnacionales son las proyecciones derivadas: aplicando a una proyección nacional previa la distribución futura en diferentes categorías, se deducen las personas pertenecientes a cada una de las categorías. La mayoría de las

\footnotetext{
* Secretaría de Programación y Presupuesto.
} 
proyecciones de la población económicamente activa y de la residente en áreas urbanas y rurales son claros ejemplos de la amplia difusión de ese procedimiento de proyección.

El método de las proyecciones derivadas, atractivo por su relativa sencillez, sobre todo cuando no se dispone de los datos necesarios para aplicar el método de las componentes, presenta sin embargo una desventaja teórica: así como la tasa de crecimiento es el producto de la dinámica demográfica pasada, la distribución de la población en diferentes categorías es resultado de la movilidad entre ellas, es decir, tal reparto es un producto y no un insumo de la dinámica poblacional.

En este artículo se presenta un método restringido de las componentes, porque no considera el sexo y la edad, para proyectar la población clasificada según el tamaño de la localidad, modificando al procedimiento utilizado en un trabajo previo (Garza y Partida, 1988). La aplicación se presenta para los núcleos urbanos (15 000 o más habitantes) de la República Mexicana en 1990, empleando la tendencia en el crecimiento demográfico para el periodo $1960-1980$.

El modelo de proyección de población según el tamaño de la localidad

Supongamos que tenemos clasificada a la población en $n$ intervalos, de acuerdo con el número de habitantes en la localidad donde reside, que en lo sucesivo llamaremos tamaño de la localidad o rango. Denotemos por $P_{i}(t)$ la población en localidades del rango i al momento $t, y$ por $C T_{i}(t, t+h)$ el factor de crecimiento total en el rango $i$ entre los momentos $t$ y $t+h$, o bien, la proporción por la que aumenta la población inicial al cabo de los $h$ años comprendidos. De estas definiciones podemos establecer que

$$
P_{i}(t+h)=P_{i}(t) \cdot C T_{i}(t, t+h)
$$

Descompongamos el crecimiento total en el natural (natalidad menos mortalidad) y en el social (inmigración menos emigración), y denotemos por $C N_{i}(t, t+h)$ y $C S_{i}(t, t+h)$ los factores de crecimiento asociados respectivamente. La extensión del método de las componentes al caso que no se considera la edad ni el sexo se puede establecer como

$$
C T_{i}(t, t+h)=C N_{i}(t, t+h) \cdot C S_{i}(t, t+h)
$$


y para los habitantes del rango i:

$$
P_{i}(\mathrm{t}+\mathrm{h})=P_{i}(\mathrm{t}) \cdot C N_{i}(\mathrm{t}, \mathrm{t}+\mathrm{h}) \cdot \mathrm{CS}_{i}(\mathrm{t}, \mathrm{t}+\mathrm{h})
$$

Basta entonces con determinar los factores de crecimiento natural y social para todos y cada uno de los tamaños de localidad, para poder obtener la población futura (al tiempo $t+h$ ). Si bien el procedimiento parece sencillo, se tiene el problema de que, al cabo del intervalo genérico de $h$ años, una o más localidades de un rango pueden cambiar a otro (superior o inferior). El método debe aplicarse entonces a cada una de las localidades de un rango específico, y el volumen de habitantes proyectado debe clasificarse en la categoría correspondiente al final del periodo.

Debido a que es difícil predecir con la suficiente precisión los valores de los factores de crecimiento natural y social para cada una de las localidades, una mejor opción es suponer que son iguales para todos los núcleos pertenecientes al mismo rango al inicio de cada periodo. Denotando por $P_{i j}(t)$ la población de la j-ésima localidad en el i-ésimo rango al inicio del periodo, el modelo de proyección (3) se puede establecer como

$$
P_{i j}(t+h)=P_{i j}(t) \cdot C N_{i}(t, t+h) \cdot C S_{i}(t, t+h)
$$

y la población resultante clasificarla en el rango correspondiente al final del periodo, pudiendo ser la misma categoría $i$ u otra. Esta última forma de proyección (4) es la que se utiliza en este artículo.

Evolución de la población de México por tamaño de la localidad, 1960-1980

En el cuadro 1 se presenta la población de la República Mexicana clasificada de acuerdo con el tamaño de la localidad en los censos de 1960, 1970 y 1980. Los doce rangos considerados corresponden al máximo número que se puede obtener al combinar los tres censos, aunque en ninguna de las publicaciones censales se ha presentado con tal amplitud. Para fines de este artículo, consideraremos como localidades urbanas a todas las zonas metropolitanas (agregación de municipios) delimitadas en 1980 por Negrete y Salazar (1986), ${ }^{1}$ así como los asentamientos humanos con 15000 o

\footnotetext{
${ }^{1}$ De las 26 zonas metropolitanas originales, a dos se les hicieron modificaciones: se agregó el municipio de Jiutepec a Cuernavaca en el estado de Morelos, y de la "Región Urbana del Bajío" sólo se consideró como una zona metropolitana
} 
más habitantes que no pertenecían a municipios metropolitanos. ${ }^{2}$ Las localidades rurales son todos los restantes núcleos reportados por los censos de población.

En las cifras del cuadro 1 se puede ver claramente el aumento en el número de localidades urbanas con el paso del tiempo, casi duplicándose al cabo de los 20 años comprendidos entre 1960 y 1980. Es notable el hecho de que, mientras el volumen de habitantes a nivel nacional aumentó $38 \%$ de 1960 a $1970,38.6$ de 1970 a 1980 y 91.4 al cabo de las dos décadas, la población residente en áreas urbanas se incrementó 65.6, 57.8 y $161.4 \%$, respectivamente.

Para la proyección motivo de este artículo, además de las localidades urbanas se consideraron aquellas que en cualquiera de los censos de 1960, 1970 o 1980 tuvieron entre 10000 y 15000 habitantes y no pertenecian a municipios metropolitanos (Partida, 1990b). En el cuadro 2 se presenta la clasificación de esas 374 localidades de acuerdo con el rango al que pertenecían al inicio y final de las dos décadas del periodo 1960-1980.

Mientras 46 de los 255 (18\%) asentamientos no urbanos en 1960 pasaron a serlo al cabo del primer decenio, sólo 14 de 209 $(6.7 \%)$ experimentaron esa transición en la década siguiente. Es notable que, mientras en la primera década ninguna localidad urbana dejó de serlo, en el periodo 1970-1980 dos pasaron a ser no urbanas, una bajando sólo un rango (Santa Bárbara, Chihuahua, de 16978 a 14 894) y la otra hasta tres (Venustiano Carranza, Chiapas, de 23624 a 8546$)^{3}$

Casi todas las 374 localidades consideradas aumentaron su población al cabo de las dos décadas ( 354 o $95 \%$ y 363 o $97 \%$, respectivamente); en cambio, 206 y 282 (55 y 75.4\%) aumentaron de rango, apenas 6 y 3 bajaron ( 1.6 y $0.8 \%$ ), y las restantes 162 y 89 ( 43 y $24 \%$ ) se mantuvieron en el mismo.

Cálculo de los factores de crecimiento demográfico por rango, 1960-1990

La información demográfica para las localidades de México, incluso agrupadas en rangos según el volumen de sus habitantes, es

a los municipios de León y San Francisco del Rincón en el estado de Guanajuato (véase Partida, 1990b).

2 El corte de 15000 ha sido el más usado para el país (por ejemplo, Unikel et al., 1978).

${ }^{3}$ Es probable que al delimitar esta localidad en el censo de 1980, parte de su territorio en 1970 se haya incorporado a otro asentamiento. 


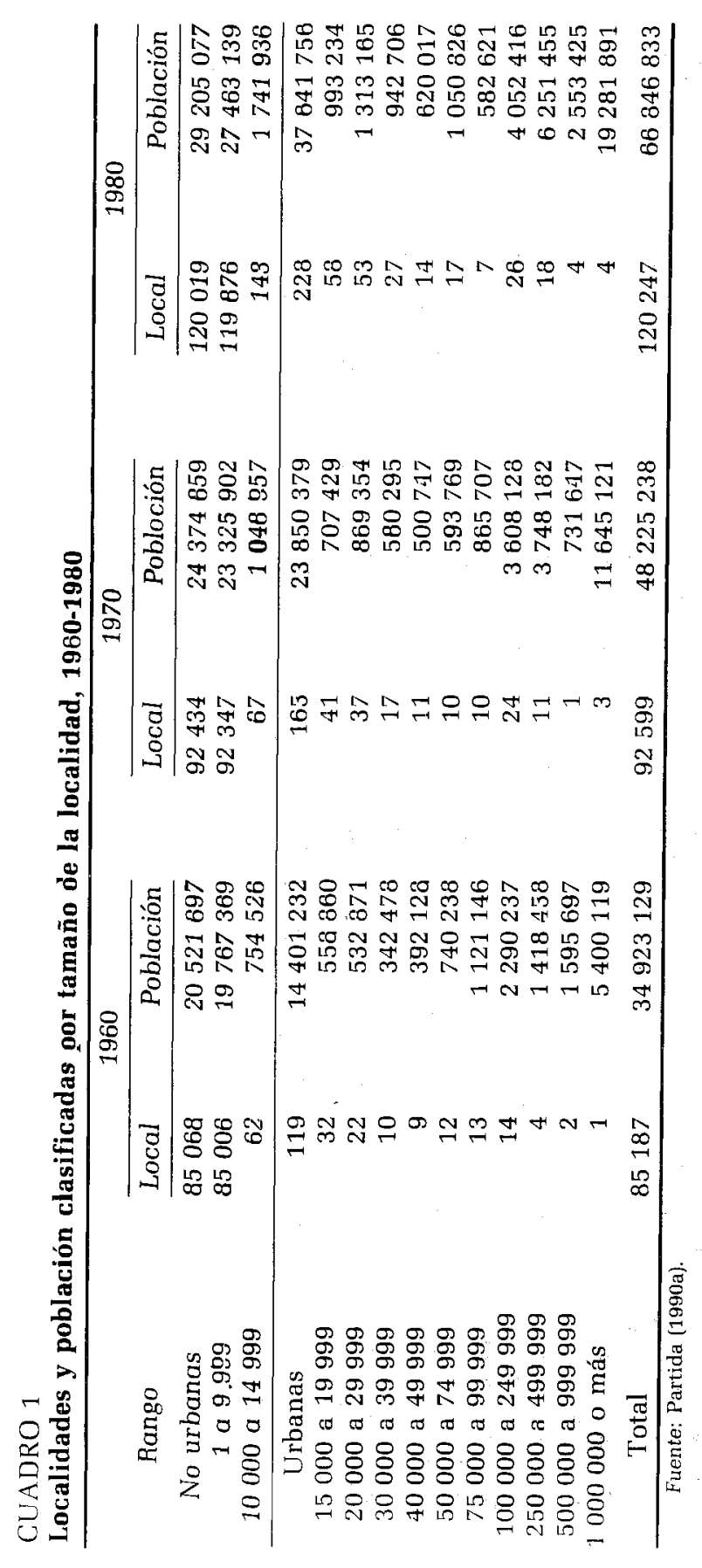




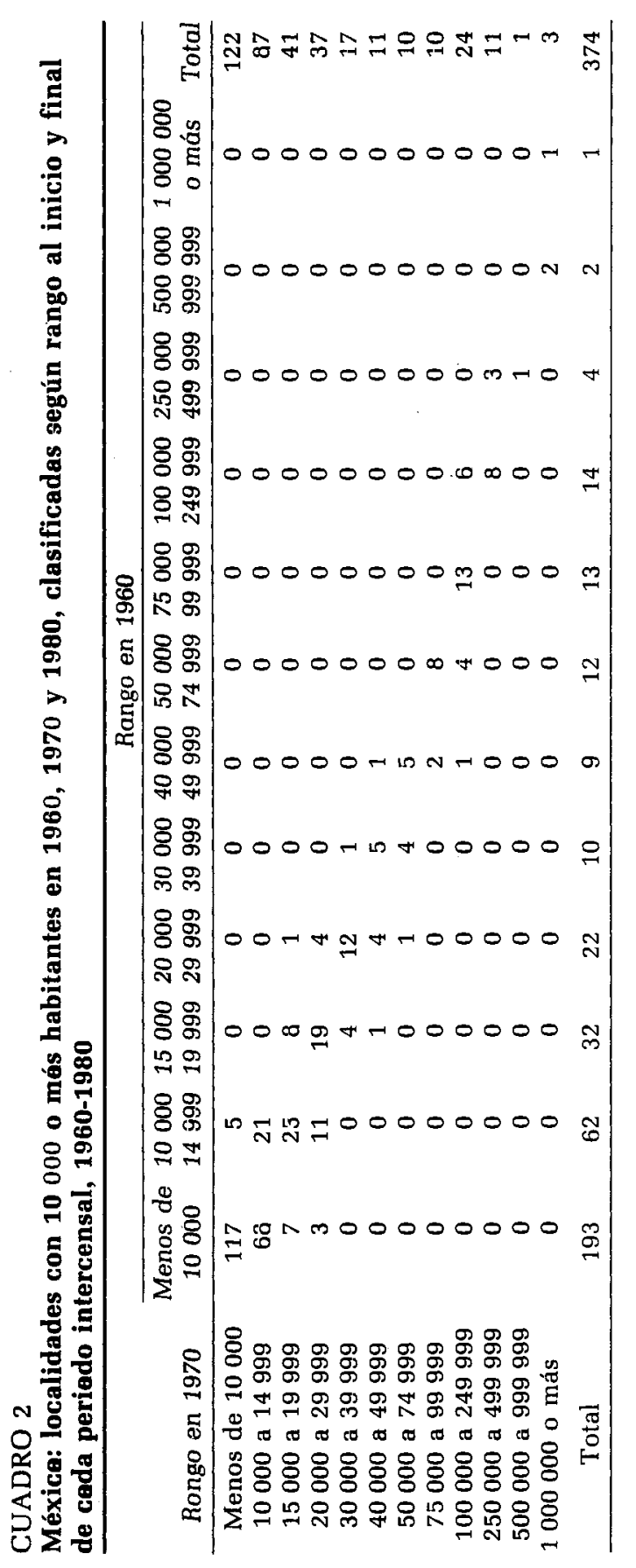




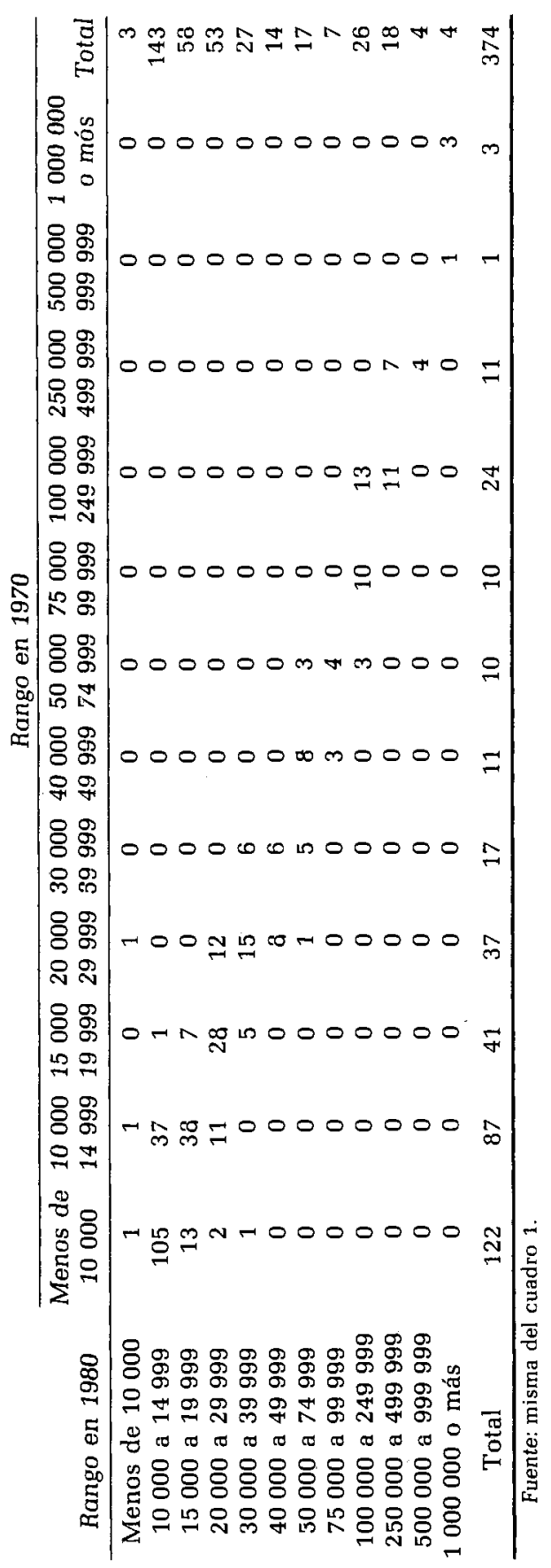


escasa: algunas estimaciones obtenidas con métodos demográficos indirectos para la fecundidad y la mortalidad urbano-rural, y prácticamente nula para el intercambio migratorio. ${ }^{4}$ Ante esta carencia de datos, creemos factible el supuesto de que, mientras debe ser reducida la brecha en el crecimiento natural entre las áreas urbanas y las rurales, debe ampliarse en el crecimiento social. Asumiremos entonces que el ritmo de crecimiento natural es igual entre las áreas urbanas y entre los asentamientos rurales, pero con un particular crecimiento social en cada núcleo. Este supuesto debe apegarse más a la realidad si en lugar de considerar localidades individuales trabajamos con su agrupación de acuerdo con el número de sus habitantes.

\section{Crecimiento natural}

Con la poca información disponible, hemos podido estimar niveles y tendencias de la fecundidad (tasa global) y la mortalidad (esperanza de vida al nacimiento por sexo) para las áreas urbanas y rurales, los cuales se reproducen en la primera parte del cuadro A.1. Convirtiendo las tasas globales de fecundidad en tasas específicas por edad, mediante el modelo de Coale y Trussel (1974), y las esperanzas de vida en probabilidades de sobrevivencia, utilizando la pauta modelo "Latinoamericano" de las Naciones Unidas (1983), hicimos una proyección por el método de las componentes sólo por crecimiento natural, esto es, asumiendo nula la ocurrencia de migraciones, para cada quinquenio del periodo 1960-1990, utilizando una proyección de población a nivel nacional previa (Partida, 1990a).

Si denotamos por $P E_{j}(t+5)$ a la población esperada en la región j (urbana, rural o nacional) por la ocurrencia exclusiva del crecimiento natural al final de un quinquenio, deducidas las pobláciones residente y esperada mediante la proyección anterior, podemos determinar los factores de crecimiento natural y social como

$$
C T_{j}(t, t,+5)=P_{j}(t+5) / P j(t) \text { y } C N_{j}(t, t,+5)=P E_{j}(t+5) / P j(t)
$$

y el de crecimiento social despejando en (2)

$$
\mathrm{CS}_{j}(\mathrm{t}, \mathrm{t}+5)=C T_{j}(\mathrm{t}, \mathrm{t}+5) / C N_{j}(\mathrm{t}, t+5)
$$

\footnotetext{
${ }^{4}$ Las estimaciones fraccionadas, con respecto a la edad y el sexo, obtenidas por Núñez y Moreno (1986: 62) son la excepción para la migración rural-urbana.
} 
Los valores correspondientes a la reconstrucción urbanorural del periodo 1960-1990 se incluyen en la segunda parte del cuadro A.1.

Si se amplía el periodo a una década, por la propiedad multiplicativa de los factores de crecimiento, los correspondientes a un intervalo decenal son

$$
\begin{aligned}
C T_{j}(t, t+10) & =C T_{j}(t, t+5) \cdot C T_{j}(t+5, t+10) \\
C N_{j}(t, t+10) & =C N_{j}(t, t+5) \cdot C N_{j}(t+5, t+10) \\
C S_{j}(t, t+10) & =C S_{j}(t, t+5) \cdot C S_{j}(t+5, t+10)
\end{aligned}
$$

que también satisfacen (2)

$$
C T_{j}[t, t+10)=C N_{j}(t, t+10) \cdot C S_{j}(t, t+10)
$$

Los factores de crecimiento decenal se presentan en la primera parte del cuadro 3.

Si bien estos factores de crecimiento natural por decenios, derivados de la reconstrucción 1960-1990, parecen ser los necesarios para nuestro objetivo, presentan dos restricciones:

i) Los intervalos intercensales no son de exactamente 10 años (9.64 años para $1960-1970,10.35$ para $1970-1980$ y 9.77 para 1980 1990).

ii) Los factores de crecimiento total obtenidos de la reconstrucción difieren de los que se pueden derivar de las cifras censales del cuadro 1 y la población nacional preliminar del censo de 1990 (cuadro 6), como se puede ver al contrastar las columnas correspondientes en la primera y tercera partes del cuadro 3.

Debido a esas limitantes, se adecuaron los factores de crecimiento natural y social deducidos de la reconstrucción, al crecimiento total desprendido de los datos censales.

Denotemos por $p$ el periodo intercensal (1 para 1960-1970, 2 para 1970-1980 y 3 para 1980-1990), y por hp la proporción que ese periodo representa del intervalo decenal respectivo (.964 para $1960-1970,1.035$ para $1970-1980$ y .977 para $1980-1990$ ); entonces los factores de crecimiento obtenidos de la reconstrucción se adecuan a los periodos intercensales como

y

$$
C T_{j}(\mathrm{p})=\left[C T_{j}(\mathrm{t}, \mathrm{t}+10)\right]^{h p} C N_{j}(\mathrm{p})=\left[C N_{j}(\mathrm{t}, \mathrm{t}+10)\right]^{h p}
$$

$$
C S_{j}(\mathrm{p})=\left[C S_{j}(\mathrm{t}, \mathrm{t}+10)\right]^{h p}
$$




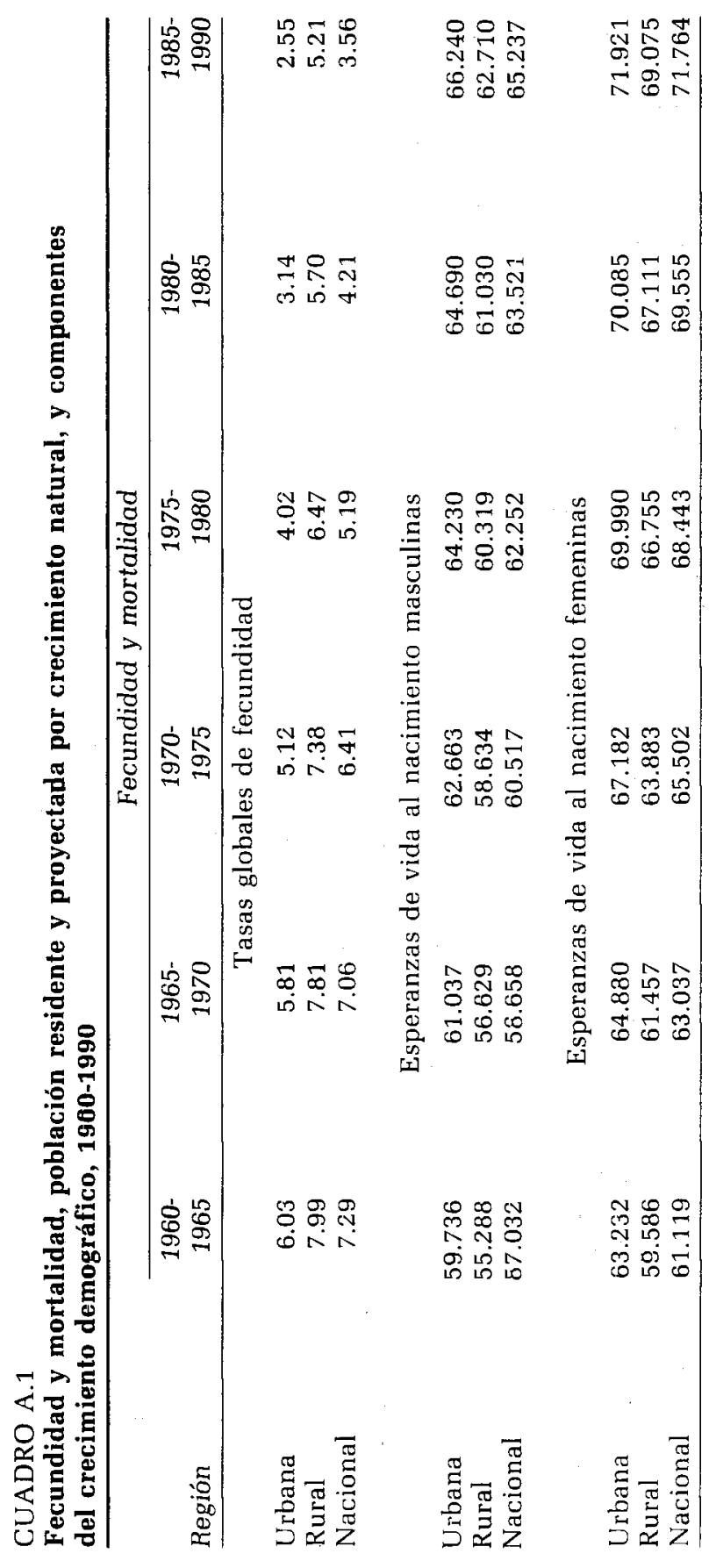




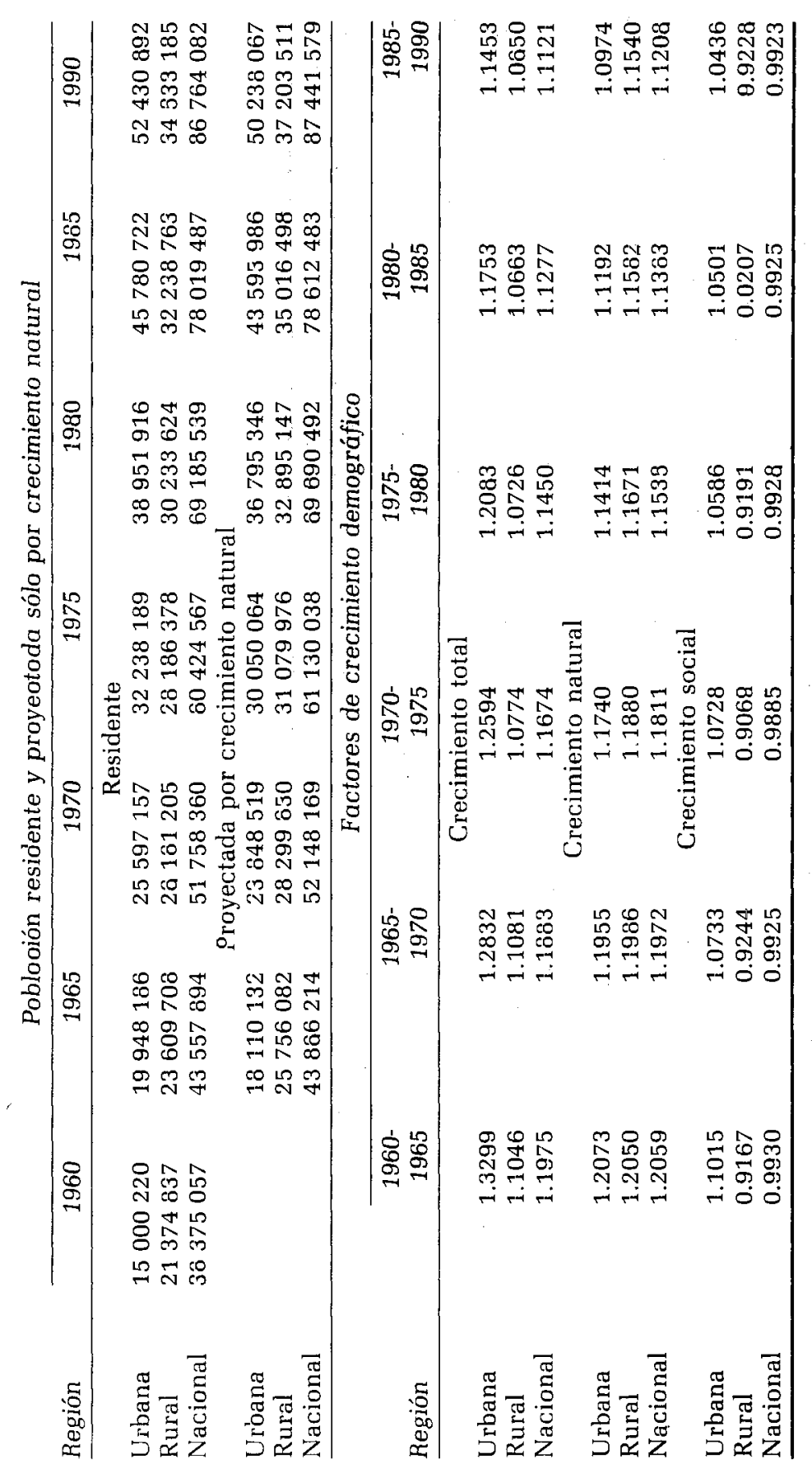




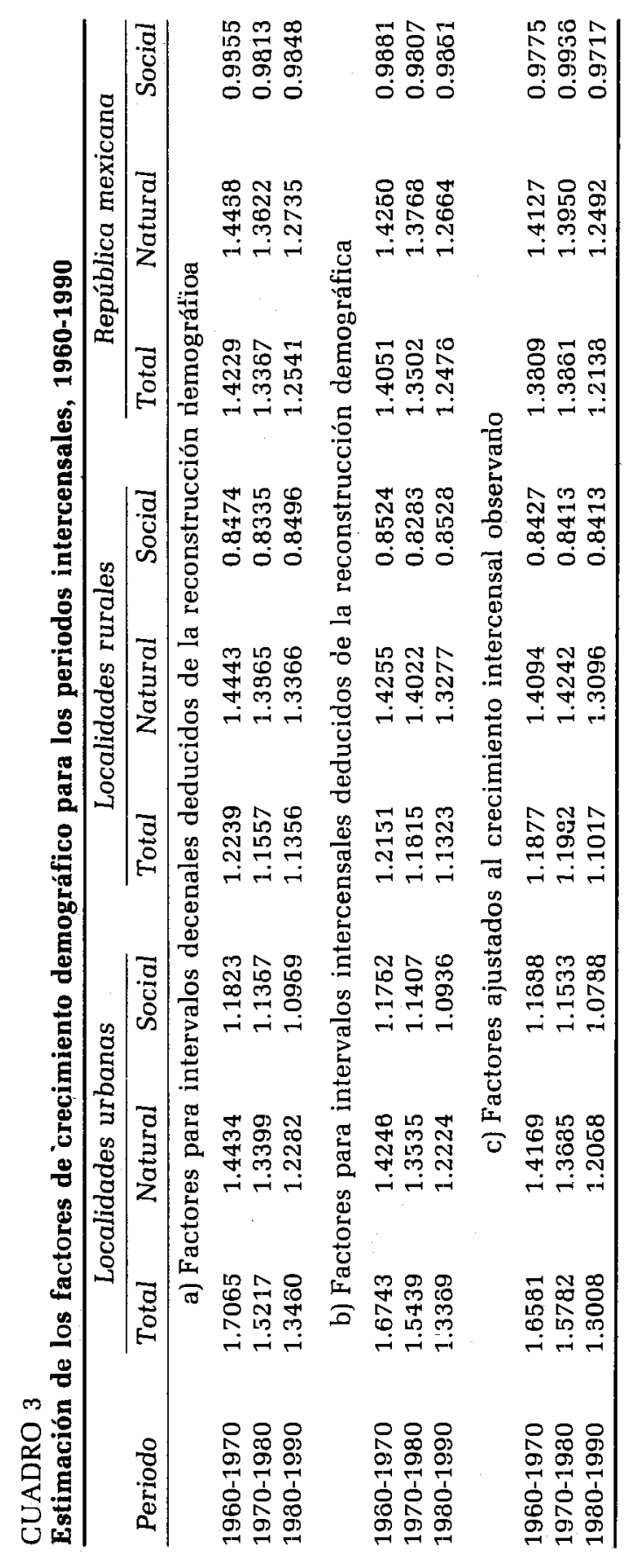


Los factores resultantes se muestran en la segunda parte del cuadro 3.

Denotemos ahora por $C T C_{j}(p), C N C_{j}(t)$ y $C S C_{j}(p)$ los factores de crecimiento total, natural y social intercensoles respectivamente, aunque con la información disponible sólo el primero se puede determinar. Introduciendo factores de corrección $w_{j}$ para los factores de crecimiento natural y social derivados de la reconstrucción demográfica:

$$
C N C_{j}(p)=w_{j} \cdot C N_{j}(p) \text { y } \operatorname{CSC}_{j}(p)=w_{j} \cdot C S_{j}(p)
$$

aplicando el principio multiplicativo (2)

$$
\operatorname{CTC}_{j}(\mathrm{t})=w_{j}{ }^{2} \cdot \mathrm{CNC}_{j}(\mathrm{p}) \cdot \mathrm{CSC}_{j}(\mathrm{p})=w_{j}{ }^{2} \cdot \mathrm{CN}_{j}(\mathrm{p}) \cdot \mathrm{CS}_{j}(\mathrm{p})=w_{j}{ }^{2} \cdot C T_{j}(\mathrm{p})
$$

de donde

$$
w_{j}=\sqrt{C T C_{j}(p) / C T_{j}(p)}
$$

Estos factores de corrección $w_{j}$ tienen la propiedad de que, en las estimaciones obtenidas para el crecimiento intercensal, mantienen el diferencial entre el crecimiento natural y social derivado de la reconstrucción demográfica:

$$
\frac{C N C_{j}(p)}{C S C_{j}(p)}=\frac{w_{j} \cdot C N_{j}(p)}{w_{j} \cdot C S_{j}(p)}=\frac{C N_{j}(p)}{C S_{j}(p)}
$$

Aplicando (6) y (5) a los datos del cuadro 3, se obtuvieron los factores de crecimiento natural y social asociados al crecimiento intercensal, para los periodos 1960-1970 y 1970-1980 en las tres regiones, y sólo a nivel nacional para el intervalo 1980-1990, debido a que en las cifras preliminares del censo de 1990 no se cuenta con las localidades según el número de habitantes, información imprescindible para clasificar los asentamientos humanos en urbanos o rurales.

Para determinar las componentes del crecimiento demográfico en la década de los ochenta, primero se estimó el crecimiento total para las áreas urbanas y rurales. Siendo urbano y rural dos subconjuntos mutuamente excluyentes y exhaustivos del territorio nacional, la población total del país en 1990 debe ser igual a la suma de los habitantes en las áreas urbanas y rurales:

$$
P_{n}(1990)=P_{u}(1990)+P_{r}(1990)
$$


e introduciendo los factores de crecimiento total para el periodo intercensal 1980-1990:

$$
P_{n}(1980) \cdot \operatorname{CTC}_{n}(3)=P_{u}(1980) \cdot \operatorname{CTC}_{u}(3)+P_{r}(1980) \cdot \operatorname{CTC}_{r}(3)
$$

Los factores desprendidos de la proyección demográfica (segunda parte del cuadro 3) no satisfacen la igualdad anterior, porque el factor de crecimiento total intercensal [CTCn(3)]difiere del derivado de la reconstrucción [CTn(3)]. Introduciendo un factor de corrección $\beta$ para los factores derivados de la proyección, igual para ambas regiones de tal manera que satisface la diferencia en el crecimiento urbano-rural

$$
\mathrm{CTC}_{u}(p)=\beta \cdot \mathrm{CT}_{u}(p) \text { y } \mathrm{CTC}_{r}(\mathrm{p})=\beta \cdot \mathrm{CT},(p)
$$

sustituyendo en (7) y despejando

$$
\beta=\frac{\operatorname{CTC}_{n}(p)}{\pi_{n}(1980) \cdot C T_{n}(3)+\pi_{r}(1980) \cdot C T_{r}(3)}
$$

donde

$$
\pi_{u}(1980)=P_{u}(1980) / P_{n}(1980) \text { y } \pi_{r}(1980)=P_{r}(1980) / P_{n}(1980)
$$

expresan la distribución urbano-rural de la población nacional.

Con los datos del cuadro 1 se determinan los valores de $\pi_{u}(1980)$ y $\pi_{r}(1980)$, y con ellos y los valores de la segunda parte del cuadro 3, aplicando (9), se obtuvo el valor de $\beta$, y aplicando (8) los factores de crecimiento intercensal 1980-1990. Utilizando (6) y (5) se estimaron los factores de crecimiento natural y social para las áreas urbanas y rurales en el intervalo 1980-1990. Los resultados se incluyen en la tercera parte del cuadro 3.

\section{Crecimiento social}

Suponiendo que el factor de crecimiento natural rural es igual para todos los núcleos en esa categoría (rangos 1 y 2), y el urbano para las localidades correspondientes (rangos 3 al 12), como establecimos con anterioridad, obtuvimos la población esperada sólo por crecimiento natural al final del intervalo intercensal:

$$
P E_{i}(t+10 \cdot h p)=P_{i}(t) \cdot C N_{i}(p) \text { con } t=1960 \text { o } 1970 \text { y } p=1 \text { o } 2(10)
$$


donde el rango i corresponde al inicio del periodo.

Clasificando los 374 núcleos considerados, de acuerdo con el rango, al principio de cada uno de los periodos intercensales 1960-1970 y 1970-1980, obtuvimos la población al inicio y final del intervalo respectivo $\left[P_{i}(\mathrm{t})\right.$ y $\left.P_{i}\left(\mathrm{t}+10^{\cdot} \mathrm{hp}\right)\right]$, volúmenes de habitantes que satisfacen (1):

$$
P_{i}(\mathrm{t}+10 \cdot \mathrm{hp})=P_{i}(\mathrm{t}) \cdot C T_{i}(\mathrm{p})
$$

y, dividiendo miembro a miembro (11) y (10), por (2) determinamos el factor de crecimiento social según el rango al inicio del intervalo respectivo $(\mathrm{p})$ :

$$
\frac{P_{i}(\mathrm{t}+10 \cdot h \mathrm{p})}{P E_{i}(\mathrm{t}+10 \cdot \mathrm{hp})}=\frac{P_{i}(\mathrm{t}) \cdot C T_{i}(\mathrm{p})}{P_{i}(\mathrm{t}) \cdot C N_{i}(\mathrm{p})}=\frac{C T_{i}(\mathrm{p})}{C N_{i}(\mathrm{p})}=C S_{i}(\mathrm{P})
$$

Los factores de crecimiento social resultante se muestran en el cuadro 4. Por ejemplo, siendo el rango 10000 a 14999 rural $(i=2)$, el factor de crecimiento natural para el periodo 1960-1970 es de 1.4094 (tercera parte del cuadro 3), y la población esperada para la fecha censal de 1970 (28 de enero) es:

$$
P E_{2}(1970)=754528 \cdot 1.4094=1063417
$$

y, como la población en 1970 de las 62 localidades en el segundo rango en 1960 fue de 933408 personas, entonces el crecimiento social para esa clasificación en el primer periodo intercensal $(p=1)$ 1960-1970 fue:

$$
\mathrm{CS}_{2}(1)=933408 / 1063417=0.9342
$$

Proyección de la población según tamaño de la localidad

El cambio en los factores de crecimiento social entre los dos primeros periodos intercensales se presenta gráficamente en la figura 1 , y de manera propocional en la tercera columna del cuadro 5. En esta última se puede ver que, excepto el intervalo 40000 a 49999 donde disminuyó casi $13 \%$, la variación entre los periodos va de $-6.3 \%$ en el undécimo rango (500 000 a 999 999) a $6.8 \%$ en el primero (menos de 10000 ).

Con base en el leve cambio proporcional en el ritmo de crecimiento social entre los dos primeros periodos intercensales, supu- 


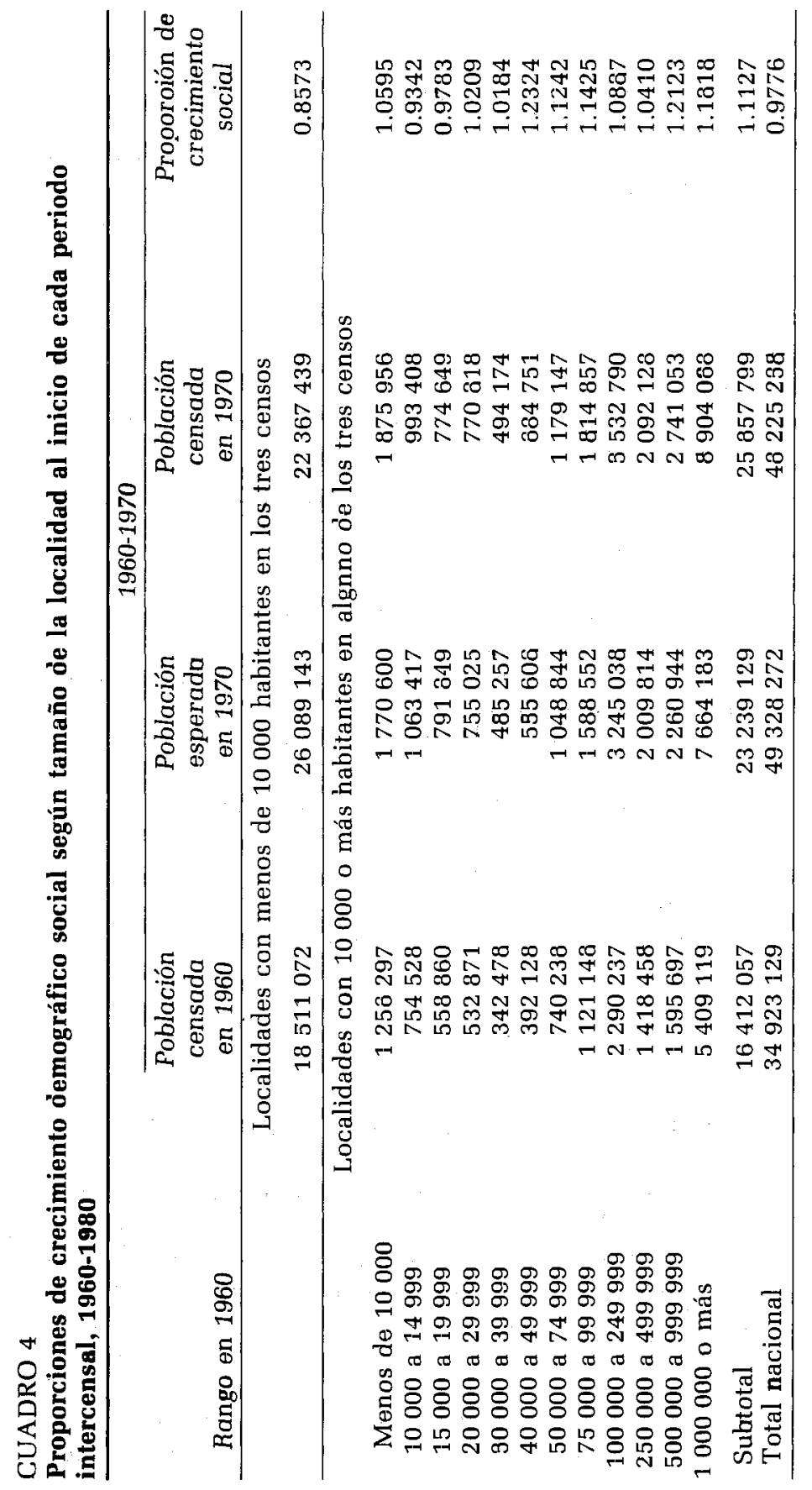




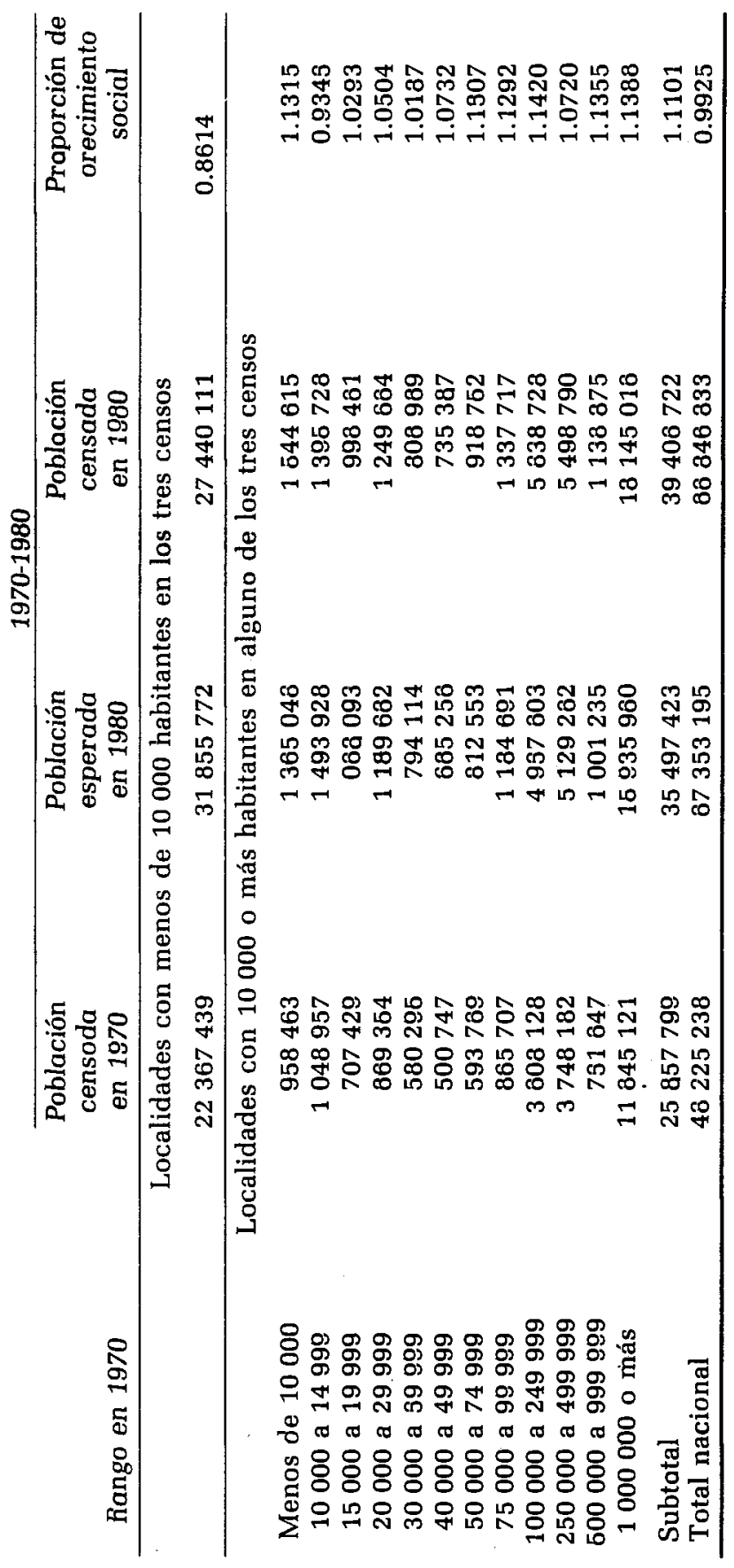


simos que para el último sería la mitad del cambio observado en los dos previos:

$$
C S_{i}(3)=C S_{i}(2) \cdot \sqrt{C S_{i}(2) / C S_{i}(1)}
$$

$y$, aplicando (2), con los factores de crecimiento natural obtenidos para el periodo 1980-1990 (último renglón de la tercera parte del cuadro 3), dedujimos los factores de crecimiento social según el rango de tamaño de la localidad en 1980 . Los resultados se muestran en las tres últimas columnas del cuadro 5.

De los factores de crecimiento total obtenidos para el periodo 1980-1990 se desprenden dos aspectos importantes:

i) Todas las localidades habrían crecido durante la década.

ii) Ningún asentamiento con menos de 10000 habitantes en 1980 habría sido urbano diez años después, pues aunque hubiera tenido el máximo de 9999 personas para el rango al incio del periodo intercensal, su población al final habría sido de 14099 $(=9999 \cdot 1.41)$

FIGURA 1

Proporciones de crecimiento social según tamaño de la localidad, 1960-1980

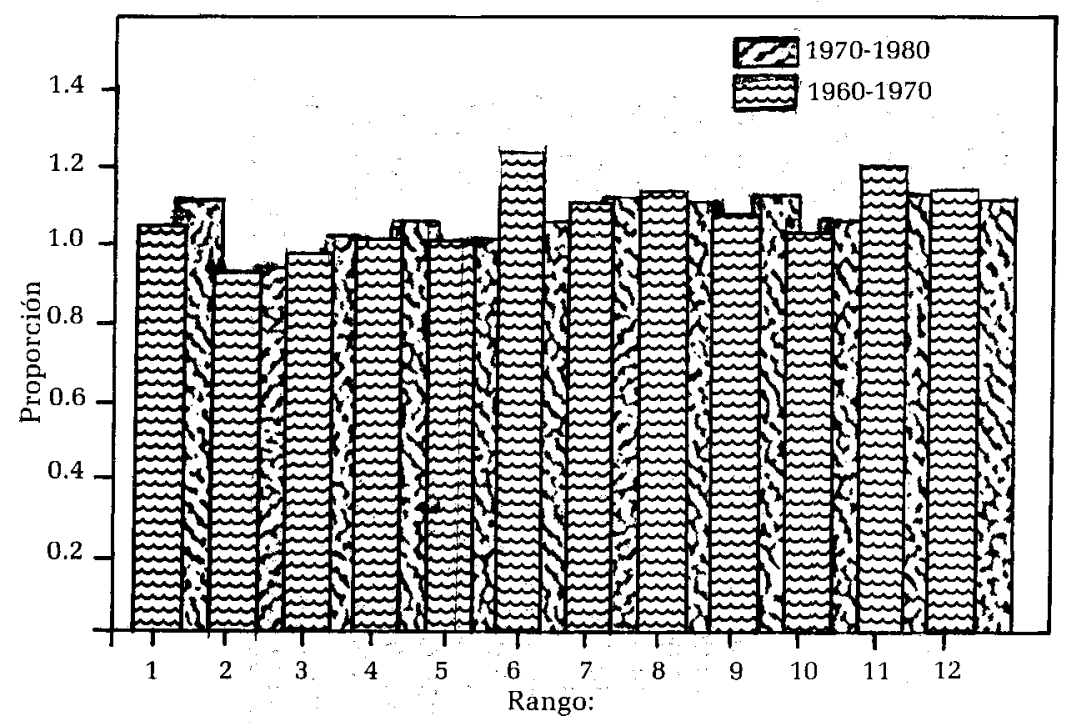

$1=$ Menos de $10000 ; 210000$ a $14999 ; \ldots 12=1000000$ o más 


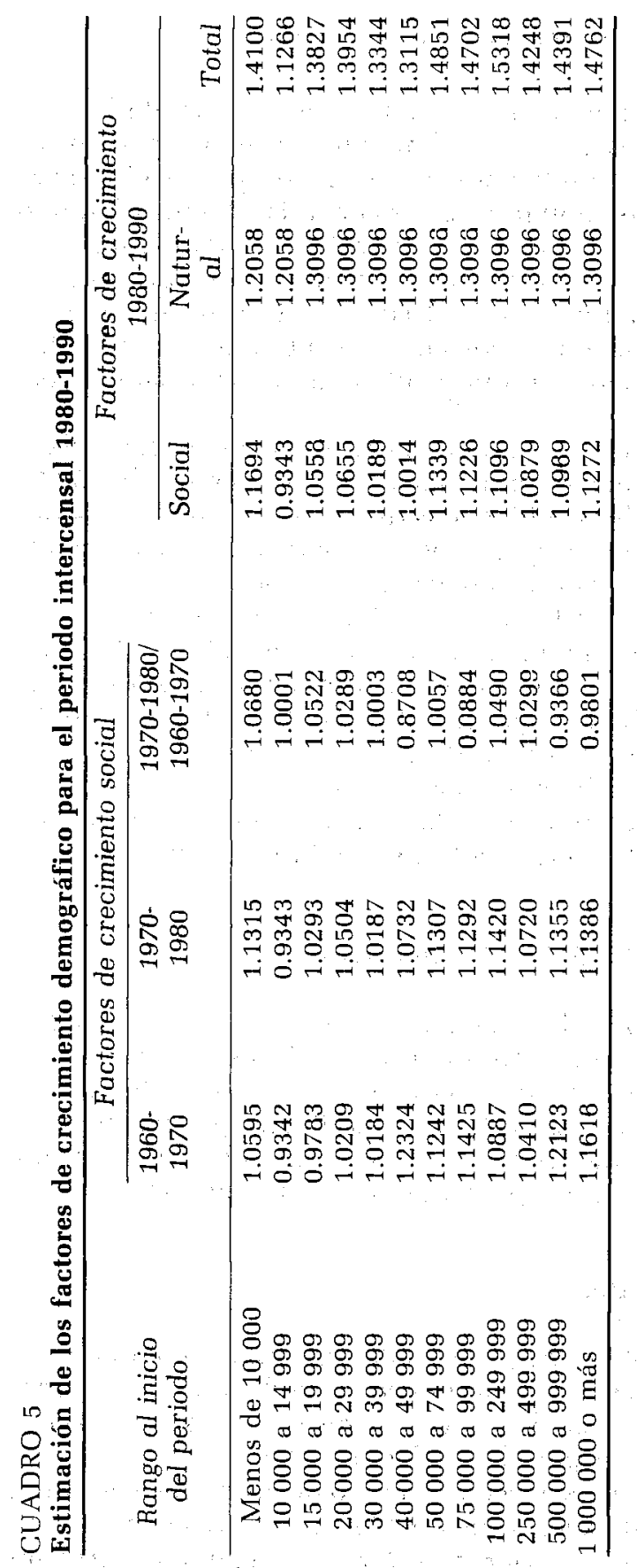


Estando nuestro interés centrado sólo en las localidades con 15000 o más habitantes (nuestra definición de urbana), dado el segundo hecho, el primero sólo actuará en nuestra proyección para los núcleos con 10000 habitantes o más en 1980. Y considerando que entre 1960 y 1970, sólo 13 de 181 (7.2\%) asentamientos con 10000 o más habitantes al inico del periodo disminuyeron su población, y entre 1970 y 1980 apenas 10 de 252 (4\%), el supuesto implícito de que todas las localidades con 10000 o más en 1980 habrían aumentado su población para 1990 parece razonablemente correcto.

El factor de crecimiento total para el primer rango (1.41), lleva implícito el supuesto que ninguna localidad con menos de 10000 habitantes en 1980 habría subido más de un rango para 1990 y por ende ser urbana, supuesto que también aparece razonablemente correcto, pues de las cifras del cuadro 2, se puede ver que para el periodo 1960-1970, entre las 374 localidades consideradas, sólo el 5.2\% (10 de 193) de los núcleos con menos de 10000 habitantes (primer rango) al inicio del periodo, pasaron más allá del rango inmediato posterior al final, y que el $13.1 \%$ (16 de 122) experimentaron el mismo tipo de transición de 1970 a 1980.

Tomando individualmente a cada una de las 371 localidades con 10000 o más habitantes en 1980 , proyectamos su población a 1990 con el factor de crecimiento total del rango a que pertenecía en 1980:

$$
P_{i j}(1990)=P_{i j}(1980) \cdot C T_{i}(3)
$$

donde $P_{i j}(1980$ o 1990) es la población de la j-ésima localidad perteneciente al i-ésimo rango en 1980 . Los valores resultantes los clasificamos entonces por rango para 1990. Los resultados se presentan en el cuadro 6, y en la figura 2 se ilustra gráficamente la tendencia temporal del porcentaje que la población urbana (15 000 o más habitantes) representa del total nacional.

\section{Consideraciones finales}

En el presente artículo se ha presentado una aplicación del método de las componentes demográficas restringido, porque en lugar de hacerlo por sexo y edad, se aplicó con los factores de crecimiento natural y social para la población total.

Los resultados de la proyección al caso de los núcleos urbanos de México para 1990 parecen razonables, como se puede ver en el cuadro 7 y la figura 3 . El incremento en las localidades urbanas 


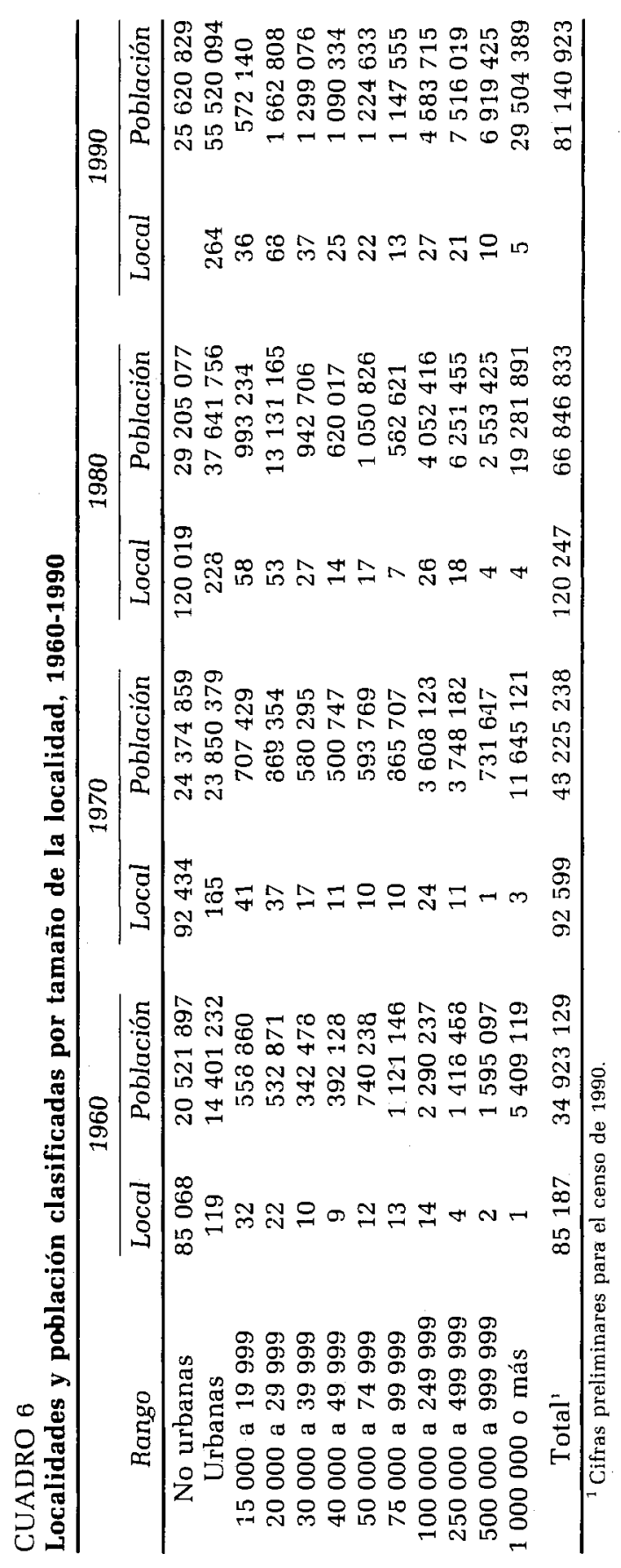


FIGURA 2

Porcentaje de la población de México residente en áreas urbanas, 1960-1990

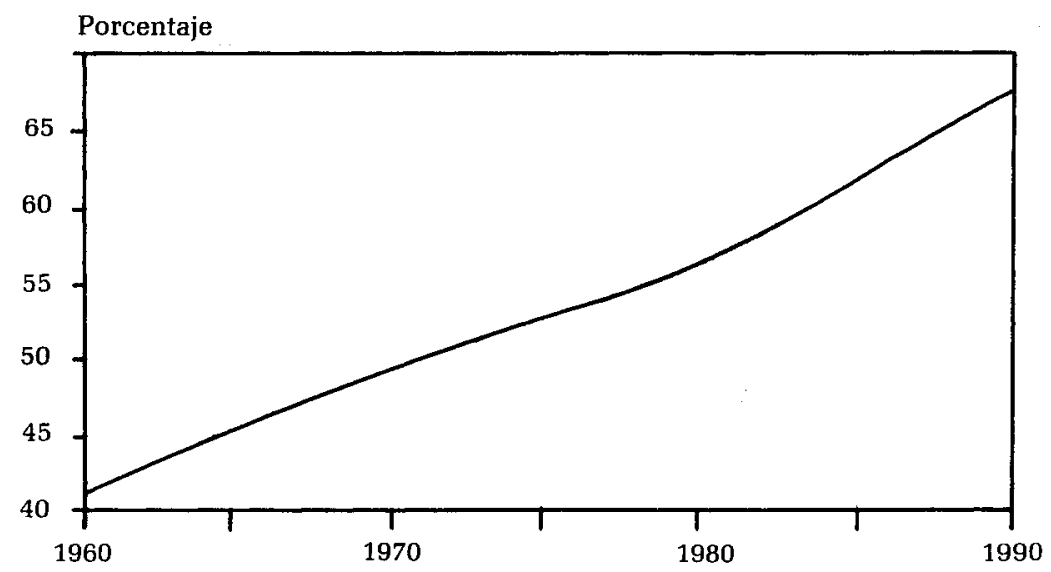

Fuente: cuadro 6

de 1980 a 1990 , dentro de cada uno de los rangos considerados, es consistente con la tendencia observada desde 1960; así como también las tasas de crecimiento demográfico (últimas tres columnas del cuadro 7), donde la disminución en prácticamente todos los rangos (la excepción es 15000 a 19999 en 1980), refleja el continuo descenso en la fecundidad (principal determinante del crecimiento natural en el país), observado en la República mexicana desde principios de la década de los setenta.

A diferencia del procedimiento de proyecciones derivadas, que utiliza como insumo un producto de la dinámica demográfica (la distribución de la población en categorías mutuamente excluyentes y exhaustivas del total nacional), el método que aquí se propone trabaja con insumos (los factores de crecimiento natural y social) y arroja como resultado la distribución de la población según tamaño de la localidad. Además permite obtener el número de asentamientos en cada rango, posibilidad que no ofrecen las proyecciones derivadas.

Basado el método en la proyección de cada una de las localidades por separado, presenta la restricción de que en la práctica es difícil obtener información demográfica al nivel de localidad, limitante que hemos superado al asumir que el ritmo de crecimiento natural fue igual dentro de los asentamientos rurales y 


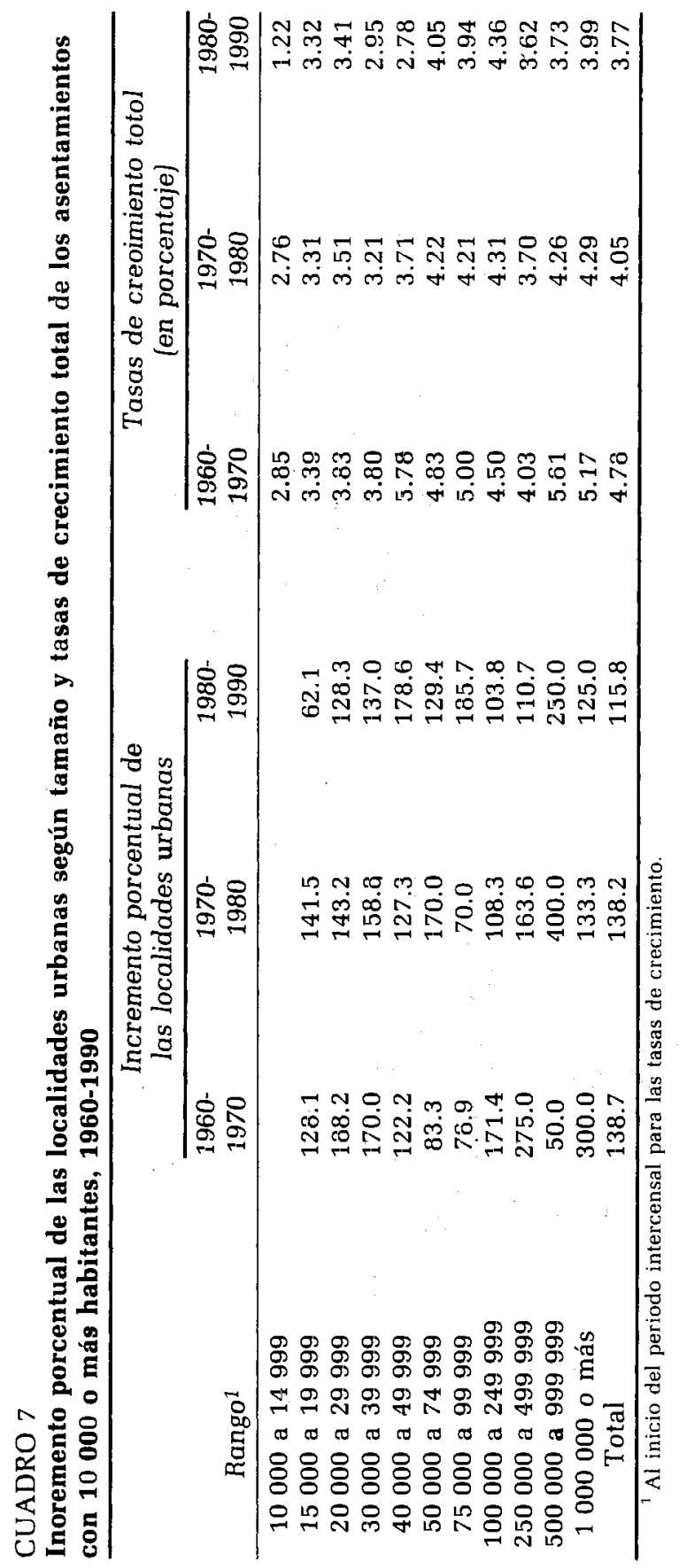




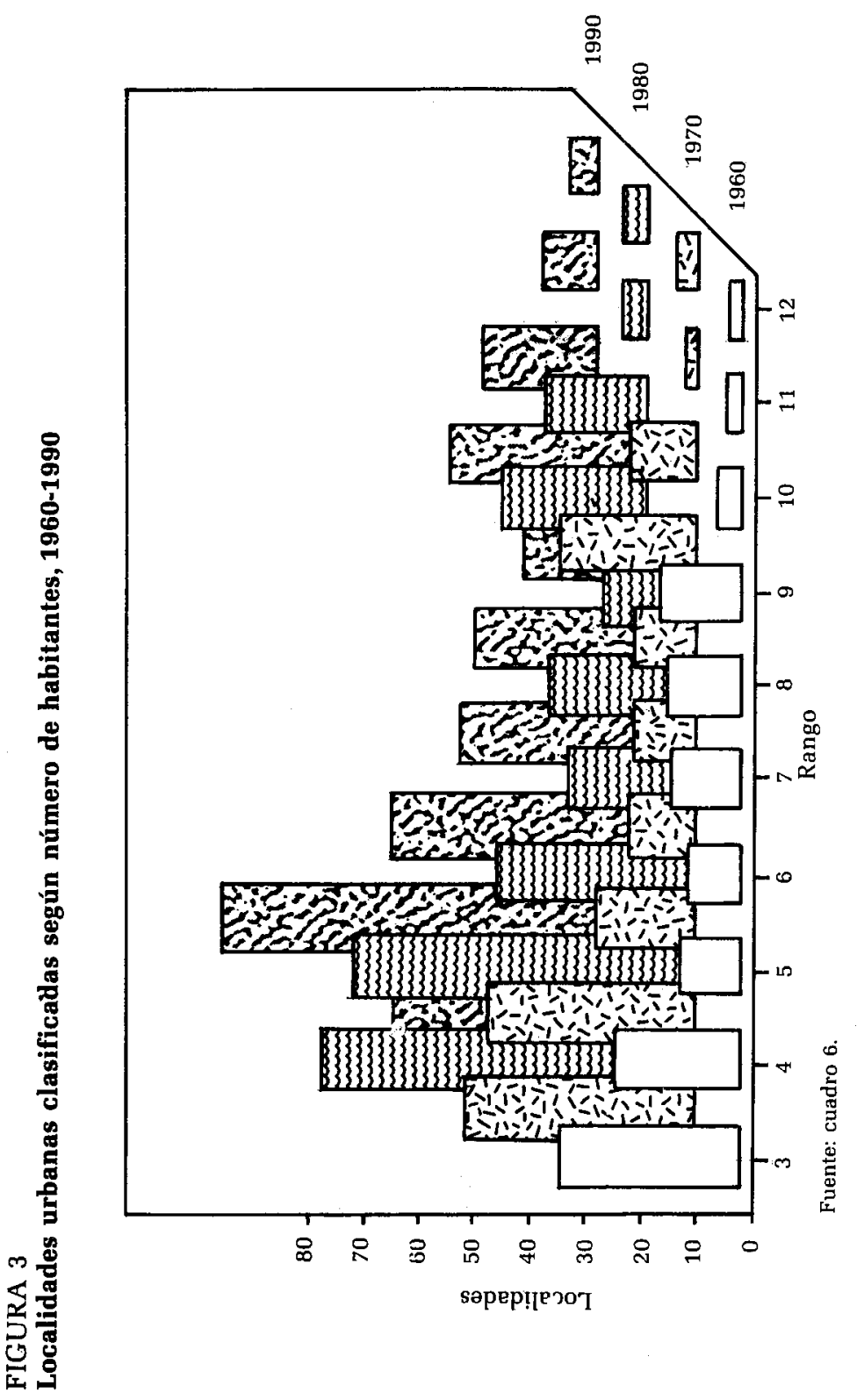


dentro de los urbanos, para los tres intervalos intercensales del periodo 1960-1990. Haber pretendido obtener sólo la proyección por grupos de localidades hace factible este supuesto; no así, si hubieramos sido tan ambiciosos como para pretender proyectar la población de cada una de las localidades.

El paso del tiempo es el mejor juez de la bondad de todo ejercicio de proyección, y dentro de unos años, cuando dispongamos de los resultados definitivos del censo de 1990 para cada localidad de la República Mexicana, nuestras estimaciones no escaparán a ese juicio evaluador.

\section{Bibliografía}

Coale, A.J. y J.T. Trussel (1974). "Model Fertility Schedules: Variations in the Age Structure of Childbearing in Human Population", en Population Index vol. 40, núm. 2, pp. 185-258.

Garza, G. y V. Partida (1988). "Desarrollo urbano. Hacia la superconcentración espacial", en Carta demográfica sobre México, Demos, México, pp. 11-12.

Naciones Unidas (1983). Tablas modelo de mortalidad para países en desarrollo, Nueva York, Naciones Unidas (sT/ESA/SER.A/77).

Negrete, M.E. y H. Salazar (1986). "Zonas metropolitanas en México, 1980", en Estudios Demográficos y Urbanos núm. 1, México, El Colegio de México, pp. 97-124.

Núñez, L. y L. Moreno (1986). México. Proyecciones de población urbana y rural 1980-2010, México, Academia Mexicana de Investigación en Demografía Médica.

Partida, V. (1990a). "El volumen, la estructura por edad y el ritmo de crecimiento de la población de México. Análisis del efecto de la dinámica demográfica y consecuencias futuras", en Revista Mexicana de Sociología, México, stunam, pp. 223-246.

(1990b). "México: Población en localidades de 10000 o más habitantes censadas en 1960, 1970 y 1980", en Estudios Demogróficos y Urbanos, en prensa.

Unikel, L., G. Garza y C. Ruiz (1978). El desarrollo urbano en México. Diagnóstico e implicaciones futuras, México, El Colegio de México. 
